\title{
Second Order Structure of Scale-Space Measurements
}

\author{
Bo Markussen • Kim Steenstrup Pedersen • Marco Loog
}

Published online: 8 April 2008

(C) Springer Science+Business Media, LLC 2008

\begin{abstract}
The second-order structure of random images $f: \mathbb{R}^{d} \rightarrow \mathbb{R}^{N}$ is studied under the assumption of stationarity of increments, isotropy and scale invariance. Scale invariance is defined via linear scale space theory. The results are formulated in terms of the covariance structure of the jet consisting of the scale space derivatives at a single point. Operators describing the effect in jet space of blurring and scaling are investigated. The theory developed is applicable in the analysis of naturally occurring images of which examples are provided.
\end{abstract}

Keywords Stationary increments - Scale invariance · Isotropy · Linear scale space · Image analysis · Covariance structure

\section{Introduction}

In this work, we consider statistical models of random functions $f: \mathbb{R}^{d} \rightarrow \mathbb{R}^{N}$ and specifically investigate linear models for their covariance structure. The investigated models

\footnotetext{
B. Markussen

Department of Natural Sciences, Faculty of Life Sciences, University of Copenhagen, Copenhagen, Denmark e-mail: bomar@life.ku.dk
}

K.S. Pedersen $(\varangle) \cdot$ M. Loog

Department of Computer Science, University of Copenhagen, Universitetsparken 1, 2100 Copenhagen, Denmark

e-mail: kimstp@diku.dk

M. Loog

e-mail: loog@diku.dk

M. Loog

Faculty of Electrical Engineering, Mathematics and Computer Science, Delft University of Technology, Delft, The Netherlands are characterized by three invariance properties: stationarity of the increments, isotropy, and scale invariance. These invariance properties are generally accepted as being useful in the analysis of natural images and supported by empirical studies [2, 3, 18, 24, 25]. However, even if such invariance assumptions would be violated to a smaller or larger extent, statistical models, as the one presented, may function very well as prior to more dedicated image analysis or processing techniques. Similarly, natural images are probably not completely characterized by their covariance structure, but still the studied class of models may act as a prior in various vision and image processing tasks. The three assumptions of stationarity, isotropy and scale invariance all restrict the covariance structure of the local jets, and further dependencies may be added in a Bayesian framework. Examples of this approach based on the Lévy Brownian model can be found in Pedersen et al. [21] which describes an image feature detection task, and Loog et al. [13] and Pedersen et al. [20] that present scale selection approaches. Furthermore, Markussen et al. [16] discuss the possibility of using the studied models for velocity fields in flow estimation.

The results presented, however, have a broader scope than image analysis. Within the field of biophysics, neurophysiology, and perception psychology, the school of ecological statistics advocates that biological sensory systems are shaped by the statistics of the stimuli caused by the habitat, and models of the type presented here, modeling the statistics of images, are very relevant (e.g. [1, 3, 24]).

The first part of this paper provides an analysis of the covariance structure of the jet of image derivatives at a single point implied by the assumptions of stationarity, isotropy, and scale invariance. In Sect. 2, we provide the definitions of these invariance properties and introduce basic notions needed in the rest of the paper. In Sect. 3, we introduce operators describing blurring, translation and scaling. In 

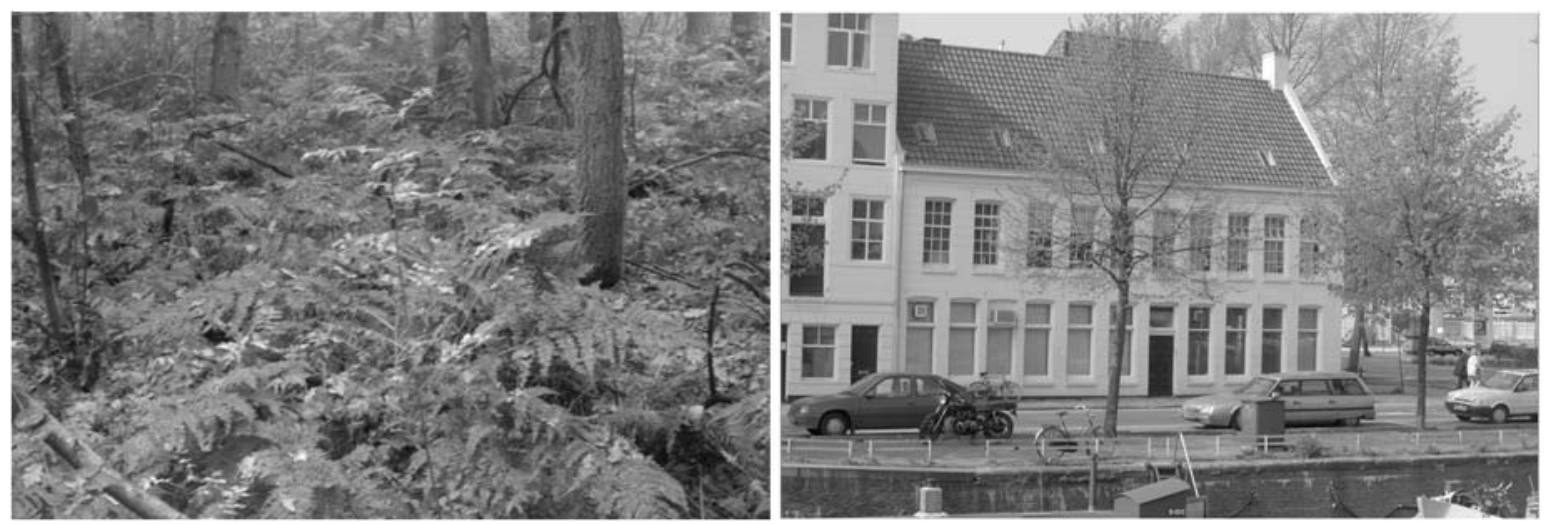

Fig. 1 A forest and a city scene taken from the van Hateren [26] database which is used in the empirical study found in Sect. 5. The resolution of these gray value images is $1024 \times 1532$ pixels

Sect. 4.1, we show that jets from images with stationary increments have a particular covariance structure with alternating signs. In Sect. 4.2, we show that the assumption of isotropy implies linear constraints within covariances of image derivatives of the same order. In Sect. 4.3, we show that the assumption of scale invariance implies linear constraints between image derivatives which differ by one order. We show that scale invariance is possible if and only if physical scale is proportional to the standard deviation in the blurring kernel. In Sect. 4.4 we use these results to give a parameterization of image models with stationary, isotropic and scale invariant increments. A sufficient condition ensuring the validity of our results is that the random function $f$ has second order moments and satisfies the mild integrability condition

$\mathrm{E}\left(\int_{\mathbb{R}^{d}} \mathrm{e}^{-\gamma\|y\|^{2}}|f(y-x)| \mathrm{d} y\right)^{2}<\infty$

for every $x \in \mathbb{R}^{d}$ and $\gamma>0$, where $\mathrm{E}$ is the expectation operator on random variables.

The second part of this paper, see Sect. 5, uses the developed theory to provide a statistical analysis of natural images such as those shown in Fig. 1. Imposing the additional assumption that the random function $f$ follows a joint Gaussian distribution our results may be employed to perform quasi-likelihood inference for a large class of image models. However, to avoid in this work the assumption of a joint Gaussian distribution we only employ moment estimators.

The results presented in this paper extends and generalizes our previous work reported in [15] in several directions. In the theoretical part we generalize to $\mathbb{R}^{N}$-valued images and include a study of isotropy. Furthermore, the Hurst index $H$ has been introduced in the definition of scale invariance (see Definition 3 in Sect. 2). In the experimental part we provide test statistics for the study of natural images, and provide new empirical results.
We would like to note that related results were also reported in early work by Longuet-Higgins [11] in a study of distributional properties of Gaussian random moving surfaces proposed as a model of ocean surface waves. LonguetHiggins results are derived based on spectral analysis in terms of moments of the power spectrum of the random function. The class of random functions considered by Longuet-Higgins have second order moments and are differentiable. From this model statistical properties on gradients and other geometrical properties are derived. LonguetHiggins [12] also provide a further development of these results by limiting to a class of isotropic random surfaces. In this paper we consider a broader class of random functions, by only requiring that they have second order moments and satisfy the integrability condition (1.1). This class include Brownian motion and fractional Brownian motion which are non-differentiable with probability 1, see e.g. Mandelbrot and van Ness [14]. We define derivatives of this class of random functions in terms of measuring with scale space derivative operators. Applying these scale space operators to Brownian and fractional Brownian motion leads to random functions which belongs to the class studied by LonguetHiggins.

In the work by Pedersen [18], the covariance structure of scale space jets of fractional Brownian images and white noise is studied and compared with that of natural images. The results of Pedersen are derived based on a spectral representation of the stochastic processes. The results presented in the present paper are derived from the three assumptions of stationarity, isotropy, and scale invariance, and is based on moment estimators.

Koenderink and van Doorn [10] studies second order differential structure of random images by building on Longuet-Higgins [11] results. Differential structure is defined via scale space derivative operators. The studied class of random functions is limited to those having a Gaussian shaped autocorrelation function. A convenient set of differential shape parameters are derived and the statistics of these 
parameters are studied on both Brownian noise images and natural images. Griffin [5] is extending the ideas found in the work of Koenderink and van Doorn in an attempt at characterizing basic local image features derivable from second order differential structure. Our work can be seen as clarifying the theoretical foundation of the random function model used by both Koenderink and van Doorn [10] and Griffin [5].

Finally we would like to mention that previously the statistical properties of another commonly used linear image representation namely the wavelet transform have been studied, especially interesting here is the studies of self-similar random processes with stationary increments $[17,22]$.

\section{Preliminary Observations, Definitions, and Remarks}

One way of describing local image structure is by means of the language of geometry and tools from differential geometry. In order to be able to apply differential geometry we have to ensure that images, i.e., functions $f: \mathbb{R}^{d} \rightarrow \mathbb{R}^{N}$ for some fixed $d, N \in \mathbb{N}$, are differentiable. A priori, this is not necessarily the case and we have to introduce some form of regularization of the differential operators.

Linear scale space theory $[4,6-8,27]$ proposes to take regularized image derivatives through the convolution with derivatives of a Gaussian kernel, i.e., via so-called Gaussian blurring. Given $s>0$ we introduce the image $f_{s}$ by

$$
\begin{aligned}
& f_{s}(x)=\int_{\mathbb{R}^{d}} g_{s}(x-y) f(y) \mathrm{d} y, \\
& g_{s}(x)=\frac{\exp \left(-\frac{x^{\top} x}{2 s}\right)}{(2 \pi s)^{d / 2}} .
\end{aligned}
$$

The usual notion of the image at scale $\sigma>0$ is given by $f_{\sigma^{2}}$. Subject to the integrability condition (1.1) the image $f_{s}$ is infinitely often differentiable with derivatives given by

$$
f_{s}^{\alpha}(x)=D_{x}^{\alpha} f_{s}(x)=(-1)^{|\alpha|} \int_{\mathbb{R}^{d}} D_{x}^{\alpha} g_{s}(x-y) f(y) \mathrm{d} y,
$$

where $D_{x}^{\alpha}=\frac{\partial^{|\alpha|}}{\partial x_{1}^{\alpha_{1} \ldots \partial x_{d}^{\alpha_{d}}}}$ and $|\alpha|=\sum_{i=1}^{d} \alpha_{i}$ for $\alpha=$ $\left(\alpha_{1}, \ldots, \alpha_{d}\right) \in \mathbb{N}_{0}^{d}$.

Local geometry and structure can be described by considering the vector of derivatives up to some order $k$, the so-called scale space $k$-jet. Traditionally, the field of image analysis has been inspired by findings in studies of the human visual system and the jet representation is, arguably [9], a biologically plausible representation of local image geometry. All partial derivatives $f_{s}^{\alpha}(x)$ are collected into the jet $\mathcal{J}_{x}\left(f_{s}\right)$ defined by

$$
\mathcal{J}_{x}\left(f_{s}\right)=\left\{f_{s}^{\alpha}(x)\right\}_{\alpha \in \mathbb{I}} \in \mathbb{R}^{N \times \mathbb{I}}, \quad \mathbb{I}=\mathbb{N}_{0}^{d} \backslash\{(0, \ldots, 0)\} .
$$

The zeroth order term is discarded as it does not carry any geometrical information. Furthermore, some of the following results are invalid if this term is included. Since only the increments $f(x)-f(y)$ are considered, we can assume without loss of generality that $f(0)=0$ and $E[f(x)]=0$.

The covariance between two random vectors $X$ and $Y$ is denoted by

$\operatorname{Cov}(X, Y)=E\left((X-E X)^{\top}(Y-E Y)\right)$,

and the variance matrix is denoted by $\operatorname{Var}(X)=\operatorname{Cov}(X, X)$.

Definition 1 (Stationarity) A random function $f$ has stationary increments $f(x)-f(y)$ if there exists a function $\rho: \mathbb{R}^{d} \rightarrow \mathbb{R}^{N \times N}$ such that $\operatorname{Var}(f(x)-f(y))=\rho(x-y)$.

Definition 2 (Isotropy) The covariance function $\rho$ is isotropic if there exists a function $\phi: \mathbb{R}_{+} \rightarrow \mathbb{R}^{N \times N}$ such that $\rho(x)=\phi(|x|)$.

Gaussian blurring can be interpreted as defocussing, i.e. only features on coarser scales than the current are recognizable. To fully implement scaling, and not merely blurring, the argument space $\mathbb{R}^{d}$ should also be appropriately scaled. The scaling of an image is characterized by the Hurst index $H>0$, and denoting by $\kappa>0$ the exponent connecting blurring scale $s$ to physical scale, we have $D_{x}^{\alpha} f_{s}\left(s^{\kappa} x\right)=$ $s^{\kappa|\alpha|} f_{s}^{\alpha}\left(s^{\kappa} x\right)$. Combining these effects we propose the following definition.

Definition 3 (Scale invariance) The covariance function $\rho$ is scale invariant if the following identity holds for every $s, t>0$ and $\alpha, \beta \in \mathbb{I}$,

$$
\begin{aligned}
& s^{\kappa(|\alpha|+|\beta|)-H} \operatorname{Cov}\left(f_{s}^{\alpha}(0), f_{s}^{\beta}(0)\right) \\
& \quad=t^{\kappa(|\alpha|+|\beta|)-H} \operatorname{Cov}\left(f_{t}^{\alpha}(0), f_{t}^{\beta}(0)\right) .
\end{aligned}
$$

To complete this section we introduce some matrix and multi-index notation used in the remaining of this paper.

Let $I_{d}, I_{N}$ and $I_{\mathbb{I}}$ be the identity operators on $\mathbb{R}^{d}, \mathbb{R}^{N}$ and $\mathbb{R}^{\mathbb{I}}$, respectively. The product $C=A B$ of two matrices $A=\left\{a_{i j}\right\}_{i, j \in \mathbb{K}}$ and $B=\left\{B_{i j}\right\}_{i, j \in \mathbb{K}}$ indexed by a general index set $\mathbb{K}$ has elements $c_{i j}=\sum_{k \in \mathbb{K}} a_{i k} b_{k j}$. The Kronecker tensor product $C=A \otimes B$ of two matrices $A=$ $\left\{a_{i j}\right\}_{i, j=1, \ldots, N} \in \mathbb{R}^{N \times N}$ and $B=\left\{b_{k l}\right\}_{k, l \in \mathbb{I}} \in \mathbb{R}^{\mathbb{I} \times \mathbb{I}}$ is the matrix with index set $\mathbb{K}=N \times \mathbb{I}$ and elements $c_{(i, k),(j, l)}=$ $a_{i j} \cdot b_{k l}$. In the scalar case $N=1$ the tensor product reduces to the usual scalar product.

For $\alpha, \beta \in \mathbb{N}_{0}^{d}$ the sum $\alpha+\beta \in \mathbb{N}_{0}^{d}$ and the difference $\alpha-$ $\beta \in \mathbb{Z}^{d}$ are defined coordinate wise. That $\alpha$ is even means that every coordinate $\alpha_{i}$ is even, $\beta \leq \alpha$ means $\beta_{i} \leq \alpha_{i}$ for 
every $i=1, \ldots, d$, and we define

$|\alpha|=\sum_{i=1}^{d}\left|\alpha_{i}\right|, \quad \alpha !=\prod_{i=1}^{d} \alpha_{i} !, \quad x^{\alpha}=\prod_{i=1}^{d} x_{i}^{\alpha_{i}}$.

\section{Gaussian Blurring in Jet Space}

In this section we study how Gaussian blurring operates on jet space. Let the operators $A_{s}, T_{x}, S_{s}: \mathbb{R}^{\mathbb{I}} \rightarrow \mathbb{R}^{\mathbb{I}}$ and the anti-diagonals $\Psi_{2 \alpha}$ be defined by

$$
\begin{array}{ll}
A_{s}=\left\{1_{\alpha \leq \beta, \beta-\alpha \operatorname{even}} \frac{(s / 2)^{\frac{|\beta-\alpha|}{2}}}{\left(\frac{|\beta-\alpha|}{2}\right) !}\right\}_{\alpha, \beta \in \mathbb{I}} \in \mathbb{R}^{\mathbb{I} \times \mathbb{I},}, \quad s \in \mathbb{R}, \\
T_{x}=\left\{1_{\alpha \leq \beta} \frac{x^{\beta-\alpha}}{(\beta-\alpha) !}\right\}_{\alpha, \beta \in \mathbb{I}} \in \mathbb{R}^{\mathbb{I} \times \mathbb{I}}, & x \in \mathbb{R}^{d}, \\
S_{S}=\left\{1_{\alpha=\beta} s^{|\alpha|}\right\}_{\alpha, \beta \in \mathbb{I}} \in \mathbb{R}^{\mathbb{I} \times \mathbb{I}}, & s>0, \\
\Psi_{2 \alpha}=\left\{(-1)^{\frac{|\beta-\gamma|}{2}} 1_{\beta+\gamma=2 \alpha}\right\}_{\beta, \gamma \in \mathbb{I}} \in \mathbb{R}^{\mathbb{I} \times \mathbb{I},} & \alpha \in \mathbb{I} .
\end{array}
$$

The basic properties of these operators are collected in the following proposition.

Proposition 1 The operators $A_{s}, T_{x}$ constitute an additive group, and the operators $S_{S}$ constitute a multiplicative group. The identities are given by $A_{0}=T_{0}=S_{1}=I_{\mathbb{I}}$, and the group operations are given by matrix multiplication, i.e.

$A_{s+t}=A_{s} A_{t}, \quad T_{x+y}=T_{x} T_{y}, \quad S_{s t}=S_{s} S_{t}$.

Furthermore, we have the commutation relations

$$
\begin{array}{ll}
A_{t} S_{s}=S_{s} A_{s^{2} t}, & T_{x} S_{s}=S_{s} T_{s y}, \\
A_{s} \Psi_{2 \alpha}=\Psi_{2 \alpha} A_{s}^{\top}, & T_{x} \Psi_{2 \alpha}=\Psi_{2 \alpha} T_{-x}^{\top} .
\end{array}
$$

In particular, $T_{x} \Psi_{2 \alpha} T_{x}^{\top}=\Psi_{2 \alpha}$ and

$$
\begin{aligned}
A_{s} \Psi_{2 \alpha} A_{s}^{\top} & =A_{2 s} \Psi_{2 \alpha} \\
& =\sum_{\beta \in \mathbb{I}: \beta \leq \alpha}(-1)^{|\alpha-\beta|} \frac{s^{|\alpha-\beta|}}{(\alpha-\beta) !} \Psi_{2 \beta} .
\end{aligned}
$$

The operators $A_{s}$ and $T_{x}$ encode blurring and translation in jet space, i.e.

$$
\begin{aligned}
& \mathcal{J}_{x}\left(f_{s+t}\right)=\left(I_{N} \otimes A_{t}\right) \mathcal{J}_{x}\left(f_{s}\right), \\
& \mathcal{J}_{x+y}\left(f_{s}\right)=\left(I_{N} \otimes T_{y}\right) \mathcal{J}_{x}\left(f_{s}\right) .
\end{aligned}
$$

In particular, deblurring is given by multiplication with $\left(I_{N} \otimes A_{t}\right)^{-1}=I_{N} \otimes A_{-t}$. Furthermore, in the scalar case $N=1$ the tensor product with $I_{N}$ can be removed.
Proof The partial derivatives $f_{s+t}^{\alpha}(x)=D_{x}^{\alpha}\left(g_{t} * f_{s}\right)(x)=$ $\left(g_{t} * f_{s}^{\alpha}\right)(x)$ with $t>0$ are given by

$$
\begin{aligned}
f_{s+t}^{\alpha}(x)= & \int_{\mathbb{R}^{d}} \frac{\exp \left(-\frac{1}{2 t}(x-y)^{\top}(x-y)\right)}{(2 \pi t)^{d / 2}} f_{s}^{\alpha}(y) \mathrm{d} y \\
= & \int_{\mathbb{R}^{d}} \frac{\exp \left(-\frac{1}{2 t}(x-y)^{\top}(x-y)\right)}{(2 \pi t)^{d / 2}} \\
& \times \sum_{\beta \in \mathbb{N}_{0}^{d}} f_{s}^{\alpha+\beta}(x) \frac{(y-x)^{\beta}}{\beta !} \mathrm{d} y \\
= & \sum_{\beta \in \mathbb{N}_{0}^{d}} \frac{f_{s}^{\alpha+\beta}(x)}{\beta !} \\
& \times \int_{\mathbb{R}^{d}} \frac{\exp \left(-\frac{1}{2 t}(x-y)^{\top}(x-y)\right)}{(2 \pi t)^{d / 2}}(y-x)^{\beta} \mathrm{d} y \\
= & \sum_{\beta \in \mathbb{N}_{0}^{d}} f_{s}^{\alpha+2 \beta}(x) \frac{t^{|\beta|}}{(2 \beta) !} \int_{\mathbb{R}^{d}} \frac{\exp \left(-\frac{y^{\top} y}{2}\right)}{(2 \pi)^{d / 2}} y^{2 \beta} \mathrm{d} y \\
= & \sum_{\beta \in \mathbb{N}_{0}^{d}} f_{s}^{\alpha+2 \beta}(x) \frac{t^{|\beta|}}{2|\beta| \beta !} .
\end{aligned}
$$

This proves the first part of (3.2) for $t>0$. Being upper triangular the matrix $A_{t}$ for $t>0$ is invertible. The convolution property $g_{s} * g_{t}=g_{s+t}$ implies the semi group property $A_{s} A_{t}=A_{s+t}$. Algebraically, this gives the inverse matrix $A_{t}^{-1}=A_{-t}$. Since the matrix $A_{t}$ is defined for every $t \in \mathbb{R}$, we have the group property $A_{s} A_{t}=A_{s+t}$ with the identity given by $A_{0}$. The analysis of the translation $T_{x}$ and scaling $S_{S}$ operators is even simpler and is left to the reader. The stated commutation relations between $A_{s}, T_{x}$ and $S_{S}$ can be found by direct matrix computations, and are in agreement with the interpretation of the operators. Similarly, we find $T_{x} \Psi_{2 \alpha}=\Psi_{2 \alpha} T_{-x}^{\top}$ and

$$
A_{s} \Psi_{2 \alpha}=\sum_{\beta \in \mathbb{I}: \beta \leq \alpha}(-1)^{|\alpha-\beta|} \frac{s^{|\alpha-\beta|}}{2^{|\alpha-\beta|}(\alpha-\beta) !} \Psi_{2 \beta}
$$

In particular, $A_{s} \Psi_{2 \alpha}$ is symmetric and we have $\Psi_{2 \alpha} A_{s}^{\top}=$ $A_{s} \Psi_{2 \alpha}$ and $A_{s} \Psi_{2 \alpha} A_{s}^{\top}=A_{2 s} \Psi_{2 \alpha}$.

Proposition 1 explains our choice to parameterize the scale with the variance in the blurring kernel $g_{s}(x)$, i.e. doing this we arrive at the additive group $A_{s}, s \in \mathbb{R}$. In particular, we get a formal expression for deblurring in jet space. However, as is well-known deblurring is numerically unstable and hence we expect the operators $A_{s}$ for $s<0$ to be ill-conditioned. 


\section{Statistical Invariance Properties in Jet Space}

The following sections provide an analysis of the structure of the jet covariance implied by the assumptions of stationarity, isotropy, and scale invariance.

\subsection{Stationary Increments}

Stationarity of the image increments $f(x)-f(y)$ implies the fundamental structure of the jet covariance on which everything else in this paper relies. Particularly, it implies that the jet covariance is independent of the spatial position $x$, and we define

$$
\begin{aligned}
\Phi_{s} & =\operatorname{Var}\left(\mathcal{J}_{0}\left(f_{s}\right)\right) \\
& =\left\{\operatorname{Cov}\left(f_{s}^{\alpha}(0), f_{s}^{\beta}(0)\right)\right\}_{\alpha, \beta \in \mathbb{I}} \in \mathbb{R}^{(N \times \mathbb{I}) \times(N \times \mathbb{I}) .}
\end{aligned}
$$

Furthermore, these covariances are determined by the variances $\operatorname{Var}\left(f_{s}^{\alpha}(0)\right)$ through the alternating signs given by $\Psi_{2 \alpha}$. We have the following proposition.

Proposition 2 If the random function $f$ has finite second order moments, stationary increments and satisfies the integrability condition (1.1), then

$\operatorname{Var}\left(\mathcal{J}_{x}\left(f_{s}\right)\right)=\Phi_{s}=\sum_{\alpha \in \mathbb{I}} \operatorname{Var}\left(f_{s}^{\alpha}(0)\right) \otimes \Psi_{2 \alpha}$,

$\operatorname{Cov}\left(\mathcal{J}_{x}\left(f_{s}\right), \mathcal{J}_{x}\left(f_{t}\right)\right)=\Phi_{\frac{s+t}{2}}$.

Moreover, the covariances $\Gamma_{\alpha}(s)=\operatorname{Var}\left(f_{s}^{\alpha}(0)\right) \in \mathbb{R}^{N \times N}$ are given by the scale-space derivatives of the covariance function via

$\Gamma_{\alpha}(s)=\frac{(-1)^{1+|\alpha|}}{2} \rho_{2 s}^{2 \alpha}(0)$

and satisfy

$\Gamma_{\alpha}(t)=\sum_{\beta \in \mathbb{N}_{0}^{d}} \frac{(s-t)^{|\beta|}}{\beta !} \Gamma_{\alpha+\beta}(s), \quad s, t>0$.

Conversely, if there exist positive semidefinite matrices $\Gamma_{\alpha}(s) \in \mathbb{R}^{N \times N}$ such that (4.1) is satisfied with $\operatorname{Var}\left(f_{s}^{\alpha}(0)\right)=$ $\Gamma_{\alpha}(s)$, then

$\operatorname{Var}\left(f_{s}(x)-f_{s}(y)\right)=2 \sum_{\alpha \in \mathbb{I}}(-1)^{1+|\alpha|} \frac{(x-y)^{2 \alpha}}{(2 \alpha) !} \Gamma_{\alpha}(s)$.

If, moreover, the limit of the preceding display exists as $s \rightarrow 0$, then $f$ has stationary increments.
Proof For positions $x, y \in \mathbb{R}^{d}$ employing $f(0)=0$ yields that $\operatorname{Cov}[f(x), f(y)]=E\left[f(x) f(y)^{\top}\right]$ equals

$$
\begin{aligned}
\frac{1}{2} E & \left((f(x)-f(0))(f(x)-f(0))^{\top}\right) \\
& +\frac{1}{2} E\left((f(y)-f(0))(f(y)-f(0))^{\top}\right) \\
& -\frac{1}{2} E\left((f(x)-f(y))(f(x)-f(y))^{\top}\right),
\end{aligned}
$$

i.e. we have the covariance $\operatorname{Cov}[f(x), f(y)]=$ $\frac{\rho(x)+\rho(y)-\rho(x-y)}{2}$. Let positions $x, y \in \mathbb{R}^{d}$, scales $s, t>0$ and differentiation orders $\alpha, \beta \in \mathbb{I}$ be given. Interchanging the blurring integrals and the expectation, which is allowed due to the integrability condition (1.1), inserting the above covariance formula, using that the integrals of the differentiated blurring kernel vanish, and doing change of variables, we have that the covariance $\operatorname{Cov}\left[f_{s}^{\alpha}(x), f_{t}^{\beta}(y)\right]$ equals

$$
\begin{aligned}
\operatorname{Cov}\left(\int_{\mathbb{R}^{d}}(-1)^{|\alpha|} D_{x}^{\alpha} g_{s}(x-u) f(u) \mathrm{d} u,\right. & \\
& \left.\int_{\mathbb{R}^{d}}(-1)^{|\beta|} D_{y}^{\beta} g_{t}(y-v) f(v) \mathrm{d} v\right) \\
= & (-1)^{|\alpha|+|\beta|} \int_{\mathbb{R}^{d}} \int_{\mathbb{R}^{d}} D_{x}^{\alpha} g_{s}(x-u) D_{y}^{\beta} g_{t}(y-v) \\
& \times \frac{\rho(u)+\rho(v)-\rho(u-v)}{2} \mathrm{~d} u \mathrm{~d} v \\
= & \frac{(-1)^{1+|\alpha|+|\beta|}}{2} \\
& \times \int_{\mathbb{R}^{d}} \int_{\mathbb{R}^{d}} D_{x}^{\alpha} g_{s}(x-u) D_{y}^{\beta} g_{t}(y-v) \rho(u-v) \mathrm{d} u \mathrm{~d} v \\
= & \frac{(-1)^{1+|\alpha|}}{2} \\
& \times \int_{\mathbb{R}^{d}} \int_{\mathbb{R}^{d}} D_{x}^{\alpha} g_{s}(x-v-z) D_{v}^{\beta} g_{t}(y-v) \rho(z) \mathrm{d} z \mathrm{~d} v \\
= & \frac{(-1)^{1+|\alpha|+|\beta|}}{2} \\
= & \times \int_{\mathbb{R}^{d}} \int_{\mathbb{R}^{d}} D_{v}^{\beta} D_{x}^{\alpha} g_{s}(x-v-z) g_{t}(y-v) \rho(z) \mathrm{d} z \mathrm{~d} v \\
= & \frac{(-1)^{1+|\alpha|}}{2} \\
& \times \int_{\mathbb{R}^{d}} \int_{\mathbb{R}^{d}} D_{x}^{\alpha+\beta} g_{s}(x-v-z) g_{t}(v-y) \rho(z) \mathrm{d} z \mathrm{~d} v \\
2 & \int_{\mathbb{R}^{d}} D_{x}^{\alpha+\beta} g_{s+t}(x-y-z) \rho(z) \mathrm{d} z . \\
= &
\end{aligned}
$$

Thus, we have $\operatorname{Cov}\left(f_{s}^{\alpha}(x), f_{t}^{\beta}(y)\right)=\frac{(-1)^{1+|\beta|}}{2} \rho_{s+t}^{\alpha+\beta}(x-y)$. Modulo the sign $(-1)^{|\beta|}$, the latter expression only depends on $\alpha, \beta$ via the sum $\alpha+\beta$. Moreover, since the covariance 
function is even the right hand side of (4.2) for $x=y$ vanishes unless the coordinates of $\alpha+\beta$ are all even. Equation (4.1) is a restatement of these properties in terms of the matrices $\Phi_{s}, \Gamma_{\alpha}(s)$ and $\Psi_{2 \alpha}$. Proposition 1 implies that the covariances $\Phi_{s+t}$ and $\Phi_{s}$ are related by

$$
\begin{aligned}
\Phi_{s+t} & =\operatorname{Var}\left(\left(I_{N} \otimes A_{t}\right) \mathcal{J}_{0}\left(f_{s}\right)\right) \\
& =\left(I_{N} \otimes A_{t}\right) \Phi_{s}\left(I_{N} \otimes A_{t}\right)^{\top},
\end{aligned}
$$

and we have

$$
\begin{aligned}
& \sum_{\alpha \in \mathbb{I}} \Gamma_{\alpha}(s+t) \otimes \Psi_{2 \alpha} \\
& \quad=\Phi_{s+t}=\sum_{\alpha \in \mathbb{I}} \Gamma_{\alpha}(s) \otimes\left(A_{t} \Psi_{2 \alpha} A_{t}^{\top}\right) \\
& \quad=\sum_{\alpha \in \mathbb{I}} \Gamma_{\alpha}(s) \otimes\left(\sum_{\beta \in \mathbb{I}: \beta \leq \alpha}(-1)^{|\alpha-\beta|} \frac{t^{|\alpha-\beta|}}{(\alpha-\beta) !} \Psi_{2 \beta}\right) \\
& \quad=\sum_{\beta \in \mathbb{I}} \sum_{\gamma \in \mathbb{N}_{0}^{d}} \frac{(-t)^{|\gamma|}}{\gamma !} \Gamma_{\beta+\gamma}(s) \otimes \Psi_{2 \beta},
\end{aligned}
$$

whereby (4.3) follows. Concerning the converse statement the Taylor representation gives

$$
\begin{aligned}
& \operatorname{Var}\left(f_{s}(x)-f_{s}(y)\right) \\
& =\operatorname{Var}\left(\sum_{\alpha \in \mathbb{I}} f_{s}^{\alpha}(0) \frac{x^{\alpha}-y^{\alpha}}{\alpha !}\right) \\
& =\sum_{\alpha, \beta \in \mathbb{I}} \frac{x^{\alpha}-y^{\alpha}}{\alpha !} \frac{x^{\beta}-y^{\beta}}{\beta !} \operatorname{Cov}\left(f_{s}^{\alpha}(0), f_{s}^{\beta}(0)\right) \\
& =\sum_{\alpha, \beta \in \mathbb{I}} 1_{\alpha+\beta \text { even }}(-1)^{\frac{|\alpha-\beta|}{2}} \Gamma_{\frac{\alpha+\beta}{2}}(s) \frac{x^{\alpha}-y^{\alpha}}{\alpha !} \frac{x^{\beta}-y^{\beta}}{\beta !} \\
& =\sum_{\gamma \in \mathbb{I}} \frac{\Gamma_{\gamma}(s)}{(2 \gamma) !} \sum_{\alpha, \beta \in \mathbb{N}_{0}^{d}: \alpha+\beta=2 \gamma} \frac{(2 \gamma) !}{\alpha ! \beta !}(-1)^{\frac{|\alpha-\beta|}{2}} \\
& \times\left(x^{2 \gamma}+y^{2 \gamma}-x^{\alpha} y^{\beta}-x^{\beta} y^{\alpha}\right) \\
& =-2 \sum_{\gamma \in \mathbb{I}} \frac{\Gamma_{\gamma}(s)}{(2 \gamma) !} \\
& \times \sum_{\alpha \in \mathbb{N}_{0}^{d}: \alpha \leq 2 \gamma} \frac{(2 \gamma) !}{\alpha !(2 \gamma-\alpha) !}(-1)^{|\gamma-\alpha|} x^{\alpha} y^{2 \gamma-\alpha} \\
& =2 \sum_{\gamma \in \mathbb{I}}(-1)^{1+|\gamma|} \frac{(x-y)^{2 \gamma}}{(2 \gamma) !} \Gamma_{2 \gamma}(s),
\end{aligned}
$$

which is (4.4). The right hand side of (4.4) only depends on $(x, y)$ via the increment $x-y$. Thus, if the limit exists as $s \rightarrow 0$, then $\operatorname{Var}(f(x)-f(y))=\lim _{s \rightarrow 0} \operatorname{Var}\left(f_{s}(x)-f_{s}(y)\right)$ only depends on $(x, y)$ via $x-y$, i.e. $f$ has stationary increments.
4.2 Isotropic Increments

Isotropy of the image increments implies linear relations between the covariances $\Gamma_{\alpha}(s)$ for derivatives $\alpha$ of the same length $|\alpha|$. For $n \in \mathbb{N}_{0}$ let the sets $\mathbb{I}_{n}$ and the functions $\eta(n, s) \in \mathbb{R}^{N \times N}$ be defined by

$\mathbb{I}_{n}=\left\{\alpha \in \mathbb{N}_{0}^{d}:|\alpha|=n\right\}$,

$\eta(n, s)=\frac{1}{2^{n+\frac{d}{2}}\left(n+\frac{d}{2}-1\right) !} \int_{0}^{\infty} y^{n+\frac{d}{2}-1} \phi(\sqrt{2 s y}) \mathrm{e}^{-y / 2} \mathrm{~d} y$.

Proposition 3 If $\rho(x)=\phi(|x|)$, then the covariances $\Gamma_{\alpha}(s)$ are given by

$\Gamma_{\alpha}(s)=\sum_{\beta \in \mathbb{N}_{0}^{2}: \beta \leq \alpha} \frac{(-1)^{1+|\alpha-\beta|}}{2^{|2 \alpha|+1} s^{|\alpha|}} \frac{(2 \alpha) !}{\beta !(\alpha-\beta) !} \eta(|\alpha-\beta|, s)$.

If, moreover, $Z_{n, \alpha} \in \mathbb{R}$ for $\alpha \in \mathbb{I}_{n}$ and $n \in \mathbb{N}$ satisfy

$\sum_{\alpha \in \mathbb{I}_{n}} \sum_{\beta \in \mathbb{I}_{m}: \beta \leq \alpha} \frac{(2 \alpha) !}{\beta !(\alpha-\beta) !} Z_{n, \alpha}=0, \quad m=0,1, \ldots, n$,

then

$\sum_{\alpha \in \mathbb{I}_{n}} Z_{n, \alpha} \Gamma_{\alpha}(s)=0, \quad s>0$.

Proof Let $H_{2 s}^{2 \alpha}(x)$ be the Hermite polynomial defined by $D_{x}^{2 \alpha} g_{2 s}(x)=H_{2 s}^{2 \alpha}(x) g_{2 s}(x)$, i.e.

$H_{2 s}^{2 \alpha}(x)=\sum_{\beta \in \mathbb{N}_{0}^{2}: \beta \leq \alpha}(-1)^{|\beta|} \frac{(2 \alpha) !}{\beta !(2 \alpha-2 \beta) !} \frac{x^{2 \alpha-2 \beta}}{2^{|2 \alpha|} S^{|2 \alpha-\beta|}}$.

Invoking a random variable $X \sim \mathcal{N}_{d}(0,2 s)$ and (4.2) we have the representation

$$
\begin{aligned}
\Gamma_{\alpha}(s) & =\frac{(-1)^{1+|\alpha|}}{2} \rho_{2 s}^{2 \alpha}(0) \\
& =\frac{(-1)^{1+|\alpha|}}{2} \int_{\mathbb{R}^{d}} D_{x}^{2 \alpha} g_{2 s}(x) \rho(x) \mathrm{d} x \\
& =\frac{(-1)^{1+|\alpha|}}{2} E\left[H_{2 s}^{2 \alpha}(X) \phi(|X|)\right] .
\end{aligned}
$$

To compute the expectation of the Hermite polynomial we condition on $Y=\frac{|X|^{2}}{2 s}$. The random variable $Y$ follows a $\chi^{2}-$ distribution with $d$ degrees of freedom, and the conditional distribution of $X$ given $Y=y$ is the uniform distribution on the sphere of radius $\sqrt{2 s y}$. We have

$$
\frac{(2 \alpha) !}{\alpha !} s^{|\alpha|}=E\left[X^{2 \alpha}\right]=\int_{0}^{\infty} E\left[X^{2 \alpha} \mid Y=y\right] P(Y \in \mathrm{d} y)
$$




$$
\begin{aligned}
& =\int_{0}^{\infty} E\left[X^{2 \alpha} \mid Y=1\right](2 s y)^{|\alpha|} P(Y \in \mathrm{d} y) \\
& =(2 s)^{|\alpha|} E\left[X^{2 \alpha} \mid Y=1\right] E\left[Y^{|\alpha|}\right] .
\end{aligned}
$$

Thus, $E\left[X^{2 \alpha} \mid Y=1\right]=\frac{(2 \alpha) !}{2^{|\alpha|} \alpha !} \frac{1}{E\left[Y^{|\alpha|}\right]}$ and we have

$$
\begin{aligned}
& E\left[H_{2 s}^{2 \alpha}(X) \phi(|X|)\right] \\
&=\sum_{\beta \in \mathbb{N}_{0}^{2}: \beta \leq \alpha}(-1)^{|\beta|} \frac{(2 \alpha) !}{\beta !(2 \alpha-2 \beta) !} \frac{1}{2^{|2 \alpha|} s^{|2 \alpha-\beta|}} \\
& \times E\left[X^{2 \alpha-2 \beta} \phi(|X|)\right] \\
&= \sum_{\beta \in \mathbb{N}_{0}^{2}: \beta \leq \alpha}(-1)^{|\beta|} \frac{(2 \alpha) !}{\beta !(2 \alpha-2 \beta) !} \frac{1}{2^{|2 \alpha|} s^{|2 \alpha-\beta|}} \\
& \times \frac{(2 \alpha-2 \beta) !}{2^{|\alpha-\beta|}(\alpha-\beta) !} \frac{(2 s)^{|\alpha-\beta|} E\left[Y^{|\alpha-\beta|} \phi(\sqrt{2 s Y})\right]}{E\left[Y^{|\alpha-\beta|}\right]} \\
&= \sum_{\beta \in \mathbb{N}_{0}^{2}: \beta \leq \alpha} \frac{(-1)^{|\beta|}}{\left.2^{|2 \alpha|}\right|^{|\alpha|}} \frac{(2 \alpha) !}{\beta !(\alpha-\beta) !} \\
& \times \frac{E\left[Y^{|\alpha-\beta|} \phi(\sqrt{2 s Y})\right]}{E\left[Y^{|\alpha-\beta|}\right]},
\end{aligned}
$$

whereby (4.5) follows with $\eta(n, s)=E\left[Y^{n} \phi(\sqrt{2 s Y})\right] /$ $E\left[Y^{n}\right]$. Furthermore, we have

$$
\begin{aligned}
\sum_{\alpha \in \mathbb{I}_{n}} Z_{n, \alpha} \Gamma_{\alpha}(s) & \\
= & \sum_{\alpha \in \mathbb{I}_{n}} \sum_{m=0}^{n} \sum_{\beta \in \mathbb{N}_{0}^{2}:|\beta|=m, \beta \leq \alpha} \frac{(-1)^{1+n+m}}{2^{2 n+1} s^{n}} \frac{(2 \alpha) !}{\beta !(\alpha-\beta) !} \\
& \times \frac{E\left[Y^{n-m} \phi(\sqrt{2 s Y})\right]}{E\left[Y^{n-m}\right]} Z_{n, \alpha} \\
= & \sum_{m=0}^{n} \frac{(-1)^{1+n+m}}{2^{2 n+1} s^{n}} \frac{E\left[Y^{n-m} \phi(\sqrt{2 s Y})\right]}{E\left[Y^{n-m}\right]} \\
& \times\left(\sum_{\alpha \in \mathbb{I}_{n}} \sum_{\beta \in \mathbb{I}_{m}: \beta \leq \alpha} \frac{(2 \alpha) !}{\beta !(\alpha-\beta) !} Z_{n, \alpha}\right),
\end{aligned}
$$

whereby (4.6) follows.

\subsection{Scale Invariance}

In this section we investigate scale invariance, which by using the scaling operator $S_{s}$ introduced in Sect. 3 can be stated as

$$
\begin{aligned}
& s^{-H} \operatorname{Var}\left(\left(I_{N} \otimes S_{S^{\kappa}}\right) \mathcal{J}_{x}\left(f_{s}\right)\right)=t^{-H} \operatorname{Var}\left(\left(I_{N} \otimes S_{t^{\kappa}}\right) \mathcal{J}_{x}\left(f_{t}\right)\right), \\
& s, t>0 .
\end{aligned}
$$

Applying this with $t$ replaced by $s+s t$ and invoking the blurring operator $A_{s t}$, we find

$$
\begin{aligned}
\Phi_{s}= & \operatorname{Var}\left(\mathcal{J}_{x}\left(f_{s}\right)\right)=\left(\frac{s+s t}{s}\right)^{-H} \\
& \times \operatorname{Var}\left(\left(I_{N} \otimes S_{s^{\kappa}}\right)^{-1}\left(I_{N} \otimes S_{(s+s t)^{\kappa}}\right) \mathcal{J}_{x}\left(f_{s+s t}\right)\right) \\
= & (1+t)^{-H} \operatorname{Var}\left(\left(I_{N} \otimes S_{(1+t)^{\kappa}} A_{s t}\right) \mathcal{J}_{x}\left(f_{s}\right)\right) \\
= & (1+t)^{-H}\left(I_{N} \otimes S_{(1+t)^{\kappa}} A_{s t}\right) \\
& \times \operatorname{Var}\left(\mathcal{J}_{x}\left(f_{s}\right)\right)\left(I_{N} \otimes S_{(1+t)^{\kappa}} A_{s t}\right)^{\top} \\
= & (1+t)^{-H}\left(I_{N} \otimes S_{(1+t)^{\kappa}} A_{s t}\right) \\
& \times \Phi_{s}\left(I_{N} \otimes S_{(1+t)^{\kappa}} A_{s t}\right)^{\top} .
\end{aligned}
$$

Thus, a scale invariant covariance structure $\Phi_{s}$ at scale $s$ is an eigenvector under the linear mappings $\Phi \mapsto$ $(1+t)^{-H}\left(I_{N} \otimes S_{(1+t)^{\kappa}} A_{s t}\right) \Phi\left(I_{N} \otimes S_{(1+t)^{\kappa}} A_{s t}\right)^{\top}$ with eigenvalue 1. If $\Phi_{s}$ is a solution to the eigenvalue problem (4.7), then Proposition 2 and Proposition 1 give

$$
\begin{aligned}
\sum_{\alpha \in \mathbb{I}} \Gamma_{\alpha}(s) \otimes \Psi_{2 \alpha}= & \Phi_{s} \\
= & (1+t)^{-H}\left(I_{N} \otimes S_{(1+t)^{\kappa}} A_{s t}\right) \\
& \times \Phi_{s}\left(I_{N} \otimes S_{(1+t)^{\kappa}} A_{s t}\right)^{\top} \\
= & \sum_{\alpha \in \mathbb{I}}\left((1+t)^{2 \kappa|\alpha|-H}\right. \\
& \left.\times \sum_{\beta \in \mathbb{N}_{0}^{d}} \frac{(-s t)^{|\beta|}}{\beta !} \Gamma_{\alpha+\beta}(s)\right) \otimes \Psi_{2 \alpha},
\end{aligned}
$$

and hence

$$
\Gamma_{\alpha}(s)=(1+t)^{2 \kappa|\alpha|-H} \sum_{\beta \in \mathbb{N}_{0}^{d}} \frac{(-s t)^{|\beta|}}{\beta !} \Gamma_{\alpha+\beta}(s) .
$$

A solution to this equation should hold true for every $t>0$, and taking the derivative with respect to $t$ at $t=0$ we find

$$
(2 \kappa|\alpha|-H) \Gamma_{\alpha}(s)=s \sum_{\beta \in \mathbb{I}_{1}} \Gamma_{\alpha+\beta}(s) .
$$

Proposition 4 If the matrices $\Gamma_{\alpha}(s)$ satisfy the recurrence relation (4.9), then $\Phi_{s}=\sum_{\alpha \in \mathbb{I}} \Gamma_{\alpha}(s) \otimes \Psi_{2 \alpha}$ satisfies

$$
\begin{aligned}
\Phi_{s}= & (1+2 \kappa t)^{-\frac{H}{2 \kappa}}\left(I_{N} \otimes S_{\sqrt{1+2 \kappa t}} A_{s t}\right) \\
& \times \Phi_{s}\left(I_{N} \otimes S_{\sqrt{1+2 \kappa t}} A_{s t}\right)^{\top} .
\end{aligned}
$$

Especially, $\Phi_{s}$ provides a solution to the eigenvalue problem (4.7) for $\kappa=\frac{1}{2}$, and for $\kappa \neq \frac{1}{2}$ a solution does not exist to (4.7). 
Proof Iterating (4.9) $m$ times for $m \in \mathbb{N}$ we have

$$
\begin{aligned}
\Gamma_{\alpha}(s) \stackrel{(4.9)}{=} & \frac{s}{2 \kappa|\alpha|-H} \sum_{\beta \in \mathbb{I}_{1}} \Gamma_{\alpha+\beta}(s) \stackrel{(4.9)}{=} \cdots \\
\stackrel{(4.9)}{=} & \frac{s^{m}}{(2 \kappa)^{m}} \frac{\left(|\alpha|-1-\frac{H}{2 \kappa}\right) !}{\left(|\alpha|+m-1-\frac{H}{2 \kappa}\right) !} \\
& \times \sum_{\beta_{1}, \ldots, \beta_{m} \in \mathbb{I}_{1}} \Gamma_{\alpha+\sum_{i=1}^{m} \beta_{i}}(s) \\
= & \frac{s^{m}}{(2 \kappa)^{m}} \frac{\left(|\alpha|-1-\frac{H}{2 \kappa}\right) !}{\left(|\alpha|+m-1-\frac{H}{2 \kappa}\right) !} \\
& \times \sum_{\beta \in \mathbb{I}_{m}} \frac{m !}{\beta !} \Gamma_{\alpha+\beta}(s) .
\end{aligned}
$$

Using this, the right hand side of (4.8) equals

$$
\begin{aligned}
& (1+t)^{2 \kappa|\alpha|-H} \sum_{\beta \in \mathbb{N}_{0}^{d}} \frac{(-s t)^{|\beta|}}{\beta !} \Gamma_{\alpha+\beta}(s) \\
& =(1+t)^{2 \kappa|\alpha|-H} \sum_{m \in \mathbb{N}_{0}} \sum_{\beta \in \mathbb{I}_{m}} \frac{(-s t)^{m}}{\beta !} \Gamma_{\alpha+\beta}(s) \\
& \stackrel{(4.11)}{=}(1+t)^{2 \kappa|\alpha|-H} \\
& \quad \times \sum_{m \in \mathbb{N}_{0}}(-2 \kappa t)^{m} \frac{\left(|\alpha|+m-1-\frac{H}{2 \kappa}\right) !}{\left(|\alpha|-1-\frac{H}{2 \kappa}\right) ! m !} \Gamma_{\alpha}(s) \\
& =\frac{(1+t)^{2 \kappa|\alpha|-H}}{(1+2 \kappa t)^{|\alpha|-\frac{H}{2 \kappa}} \Gamma_{\alpha}(s),}
\end{aligned}
$$

where we have used the normalization identity for the negative binomial distribution. This gives

$$
\begin{aligned}
& (1+t)^{-H}\left(I_{N} \otimes S_{(1+t)^{\kappa}} A_{s t}\right) \Phi_{s}\left(I_{N} \otimes S_{(1+t)^{\kappa}} A_{s t}\right)^{\top} \\
& =\frac{(1+t)^{-H}}{(1+2 \kappa t)^{-\frac{H}{2 \kappa}}}\left(I_{N} \otimes S_{\frac{(1+t)^{\kappa}}{\sqrt{1+2 \kappa t}}}\right) \Phi_{S}\left(I_{N} \otimes S_{\frac{(1+t)^{\kappa}}{\sqrt{1+2 \kappa t}}}\right)^{\top}
\end{aligned}
$$

and hence (4.10). Finally, we recognize that there exists a solution to the eigenvalue problem (4.7) if and only if $\kappa=\frac{1}{2}$. Furthermore, $\Phi_{s}$ is an eigenvector in the affirmative case.

\subsection{Models with Stationary, Isotropic and Scale Invariant Increments}

In this section we use the results of the previous sections to provide a parameterization of image models with stationary, isotropic and scale invariant increments. Proposition 4 implies that $\kappa=\frac{1}{2}$ for such models.

First we provide an example of a model within this class of models. A Lévy Brownian motion with contrast parameter $\xi$ is a Gaussian process $f: \mathbb{R}^{d} \rightarrow \mathbb{R}$ with stationary increments and covariance function $\rho(x)=\xi|x|$. Clearly, the
Lévy Brownian motion has isotropic increments, whereby Proposition 3 implies that $s^{|\alpha|-\frac{1}{2}} \Gamma_{\alpha}(s)$ is independent of $s>0$. Thus, the Lévy Brownian motion is scale invariant with $H=\frac{1}{2}$.

Proposition 5 For every $H>0$ there exists constants $c_{\alpha, 0}, c_{\alpha, 1}, d_{n, 0}, d_{n, 1} \in \mathbb{R}$ such that every image model with stationary, isotropic and scale invariant increments (with this $H$ ) satisfies

$\Gamma_{\alpha}(s)=\frac{c_{\alpha, 0} \eta(0,1)+c_{\alpha, 1} \eta(1,1)}{s^{|\alpha|-H}}$,

$\eta(n, s)=s^{H}\left(d_{n, 0} \eta(0,1)+d_{n, 1} \eta(1,1)\right)$.

Thus, these image models are parameterized by $\eta(0,1)$, $\eta(1,1) \in \mathbb{R}$.

Proof First we show by induction that there exists $c_{\alpha, 0}, c_{\alpha, 1}$, $d_{n, 0}, d_{n, 1} \in \mathbb{R}$ such that

$\Gamma_{\alpha}(s)=\frac{c_{\alpha, 0} \eta(0, s)+c_{\alpha, 1} \eta(1, s)}{s^{|\alpha|}}$,

$\eta(n, s)=d_{n, 0} \eta(0, s)+d_{n, 1} \eta(1, s)$.

Proposition 3 implies

$$
\begin{aligned}
\Gamma_{\alpha}(s) & =\frac{\eta(1, s)-\eta(0, s)}{3 s} \\
& =\frac{\eta(1,1)-\eta(0,1)}{3 s^{1-H}}, \quad \alpha \in \mathbb{I}_{1},
\end{aligned}
$$

i.e. the above statement is true for $n=1$. Assume that it holds for all $m \leq n$. For fixed $\alpha \in \mathbb{I}_{n}$ (4.9) gives

$$
\begin{aligned}
& \sum_{\beta \in \mathbb{I}_{1}} \Gamma_{\alpha+\beta}(s) \\
& \quad=\frac{n-H}{s} \Gamma_{\alpha}(s) \\
& \quad=\frac{(n-H) c_{\alpha, 0} \eta(0, s)+(n-H) c_{\alpha, 1} \eta(1, s)}{s^{n+1}} .
\end{aligned}
$$

On the other hand (4.5) gives

$$
\begin{aligned}
\sum_{\beta \in \mathbb{I}_{1}} \Gamma_{\alpha+\beta}(s)= & \sum_{m=0}^{n+1} \sum_{\beta \in \mathbb{I}_{1}} \sum_{\gamma \in \mathbb{I}_{m}: \gamma \leq \alpha+\beta} \frac{(-1)^{n-m}}{2^{2 n+3} s^{n+1}} \\
& \times \frac{(2 \alpha+2 \beta) !}{\gamma !(\alpha+\beta-\gamma) !} \eta(n+1-m, s),
\end{aligned}
$$

which equals

$$
\sum_{\beta \in \mathbb{I}_{1}} \frac{(-1)^{n}}{2^{2 n+3} s^{n+1}} \frac{(2 \alpha+2 \beta) !}{(\alpha+\beta) !} \eta(n+1, s)
$$




$$
\begin{aligned}
& +\sum_{m=1}^{n+1} \sum_{\beta \in \mathbb{I}_{1}} \sum_{\gamma \in \mathbb{I}_{m}: \gamma \leq \alpha+\beta} \frac{(-1)^{n-m}}{2^{2 n+3} s^{n+1}} \frac{(2 \alpha+2 \beta) !}{\gamma !(\alpha+\beta-\gamma) !} \\
& \times\left(d_{n+1-m, 0} \eta(0, s)+d_{n+1-m, 1} \eta(1, s)\right) .
\end{aligned}
$$

Combining these equations we see, that $\eta(n+1, s)$ equals

$$
\begin{aligned}
& \left(\sum_{\beta \in \mathbb{I}_{1}} \frac{(-1)^{n}}{2^{2 n+3} s^{n+1}} \frac{(2 \alpha+2 \beta) !}{(\alpha+\beta) !}\right)^{-1} \\
& \quad \times\left(\frac{(n-H) c_{\alpha, 0} \eta(0, s)+(n-H) c_{\alpha, 1} \eta(1, s)}{s^{n+1}}\right. \\
& \quad-\sum_{m=1}^{n+1} \sum_{\beta \in \mathbb{I}_{1}} \sum_{\gamma \in \mathbb{I}_{m}: \gamma \leq \alpha+\beta} \frac{(-1)^{n-m}}{2^{2 n+3} s^{n+1}} \frac{(2 \alpha+2 \beta) !}{\gamma !(\alpha+\beta-\gamma) !} \\
& \left.\quad \times\left(d_{n+1-m, 0} \eta(0, s)+d_{n+1-m, 1} \eta(1, s)\right)\right),
\end{aligned}
$$

which equals $d_{n+1,0} \eta(0, s)+d_{n+1,1} \eta(1, s)$ for some constants $d_{n+1,0}, d_{n+1,1} \in \mathbb{R}$. Thereafter the constants $c_{\alpha, 0}$, $c_{\alpha, 1} \in \mathbb{R}$ for $\alpha \in \mathbb{I}_{n+1}$ are found via (4.5). This completes the induction step.

Thus, for $\alpha \in \mathbb{I}$ the definition of scale invariance implies that

$s^{|\alpha|-H} \Gamma_{\alpha}(s)=s^{-H}\left(c_{\alpha, 0} \eta(0, s)+c_{\alpha, 1} \eta(1, s)\right)$

does not depend on $s>0$. Since the constants $c_{\alpha, 0}$ and $c_{\alpha, 1}$ must depend on $\alpha$ this implies that $s^{-H} \eta(0, s)$ and $s^{-H} \eta(1, s)$ must be independent of $s>0$. This completes the proof.

\section{Moment Estimators}

The preceding sections have studied implications of stationarity, isotropy and scale invariance on the jet covariance structure. Reversing these relations we may study the invariance properties of images via the empirical covariances of the local jets. Suppose we have observed a possibly non-stationary, non-isotropic, and non-scale invariant image $\tilde{f}: \mathbb{R}^{d} \rightarrow \mathbb{R}^{N}$. Suppose there exists a stationary image $f: \mathbb{R}^{d} \rightarrow \mathbb{R}^{N}$ and a mapping $\xi: \mathbb{R}^{d} \times \mathbb{R}_{+} \rightarrow \mathbb{R}_{+}$such that

$$
\begin{aligned}
& \operatorname{Cov}\left(\mathcal{J}_{x}\left(\tilde{f}_{\xi(x, s)}\right), \mathcal{J}_{x}\left(\tilde{f}_{\xi(x, t)}\right)\right) \\
& \quad=\operatorname{Cov}\left(\mathcal{J}_{x}\left(f_{s}\right), \mathcal{J}_{x}\left(f_{t}\right)\right), \quad s, t \geq 0 .
\end{aligned}
$$

Then we have from Proposition 2

$$
\begin{aligned}
\mathrm{E} & {\left[\mathcal{J}_{x}\left(\tilde{f}_{\xi(x, s)}\right) \mathcal{J}_{x}\left(\tilde{f}_{\xi(x, t)}\right)^{\top}\right] } \\
& =\operatorname{Var}\left(\mathcal{J}_{x}\left(f_{\frac{s+t}{2}}\right)\right)=\sum_{\alpha \in \mathbb{I}} \Gamma_{\alpha}\left(\frac{s+t}{2}\right) \otimes \Psi_{2 \alpha} .
\end{aligned}
$$

Moment estimators $\widehat{\Gamma}_{\alpha}(s)$ for the covariances $\Gamma_{\alpha}(s)$ are defined by equating the empirical and the theoretical covariances, i.e.

$$
\begin{aligned}
\widehat{\Gamma}_{\alpha}\left(\frac{s+t}{2}\right)= & \frac{1}{M} \sum_{i=1}^{M} \frac{\mathcal{J}_{x_{i}}\left(\tilde{f}_{\xi\left(x_{i}, s_{i}\right)}\right) \cdot \Psi_{2 \alpha} \cdot \mathcal{J}_{x_{i}}\left(\tilde{f}_{\xi\left(x_{i}, t_{i}\right)}\right)^{\top}}{\operatorname{tr}\left(\Psi_{2 \alpha} \Psi_{2 \alpha}^{\top}\right)} \\
& \in \mathbb{R}^{N \times N}, \quad s, t \geq 0
\end{aligned}
$$

where $\mathcal{J}_{x}\left(\widetilde{f}_{\xi(x, s)}\right) \in \mathbb{R}^{N \times \mathbb{I}}, \Psi_{2 \alpha} \in \mathbb{R}^{\mathbb{I} \times \mathbb{I}}$, and the sum is taken over $M$ sample pairs at $\left(x_{i}, s_{i}\right)$ and $\left(x_{i}, t_{i}\right)$. We remark, that matrix products over $\mathbb{I}$ in (5.3) reduce to finite sums since the $(\beta, \gamma)^{\prime}$ th elements in $\Psi_{2 \alpha}$ vanish for $\beta, \gamma \geq 2 \alpha$. The assumption (5.1) can be partly checked by investigating whether the estimates $\widehat{\Gamma}_{\alpha}\left(\frac{s+t}{2}\right)$ only depend on $s$ and $t$ through $s+t$ within random variation.

Stationary increments also implies covariance relations of the form given in (5.2). To test this relation for empirical images we restrict the infinite dimensional jets $\mathcal{J}_{x}\left(\widetilde{f}_{\xi(x, s)}\right)$ to the $k$-jets

$$
\begin{gathered}
\mathcal{J}_{x}^{k}\left(\tilde{f}_{\xi(x, s)}\right)=\left\{\tilde{f}_{\xi(x, s)}^{\alpha}(x)\right\}_{\alpha \in \mathbb{I}_{\leq k}} \in \mathbb{R}^{N \times \mathbb{I}_{\leq k},} \\
\mathbb{I}_{\leq k}=\left\{\alpha \in \mathbb{N}_{0}^{d}: 1 \leq|\alpha| \leq k\right\},
\end{gathered}
$$

and construct two random variables from the covariance estimates $\widehat{\Gamma}_{\alpha}(s), \quad \alpha \in \mathbb{I}_{\leq k}$, and the random variables $\mathcal{J}_{x}^{k}\left(\widetilde{f}_{\xi(x, s)}\right)$ : The singular value decomposition (SVD) of (5.2) restricted to the $k$-jet provides unitary matrices $U, V$ and a diagonal matrix $D$ with nonnegative elements such that

$\sum_{\alpha \in \mathbb{I}_{\leq k}} \widehat{\Gamma}_{\alpha}(s) \otimes \Psi_{2 \alpha}=U D V^{\top} \in \mathbb{R}^{\left(N \times \mathbb{I}_{\leq k}\right) \times\left(N \times \mathbb{I}_{\leq k}\right)}=\mathbb{R}^{n \times n}$,

where $n$ equals $N$ multiplied by the size of $\mathbb{I}_{\leq k}$. Using this decomposition we normalize the covariances of $\mathcal{J}_{x}^{k}\left(\widetilde{f}_{\xi(x, s)}\right)$ via the random vector $Y$ defined by

$Y=U D^{-1 / 2} V^{\top} \mathcal{J}_{x}^{k}\left(\tilde{f}_{\xi(x, s)}\right)^{\top} \in \mathbb{R}^{n}$.

If the assumption of stationary increments holds, then $E[Y]=0$ and $\operatorname{Var}[Y]=I_{n}$. Thus, if $Y=\left(Y_{1}, \ldots, Y_{n}\right)$ denotes coordinates of $Y$, then

$\mathrm{E}\left[\sum_{i \neq j} Y_{i} Y_{j}\right]=0, \quad \frac{1}{n} \mathrm{E}\left[\sum_{i} Y_{i}^{2}\right]=1$.

In the limit as the number of samples $M$ goes to infinity the central limit theorem implies that the following two test statistics are asymptotically normal distributed $\mathcal{N}(0,1)$,

$$
\frac{\sum_{k=1}^{M} \sum_{i \neq j} Y_{k, i} Y_{k, j}}{\sqrt{\sum_{k=1}^{M}\left(\sum_{i \neq j} Y_{k, i} Y_{k, j}\right)^{2}}}, \quad \frac{\sum_{k=1}^{M}\left(\frac{1}{n} \sum_{i} Y_{k, i}^{2}-1\right)}{\sqrt{\sum_{k=1}^{M}\left(\frac{1}{n} \sum_{i} Y_{k, i}^{2}-1\right)^{2}}} .
$$



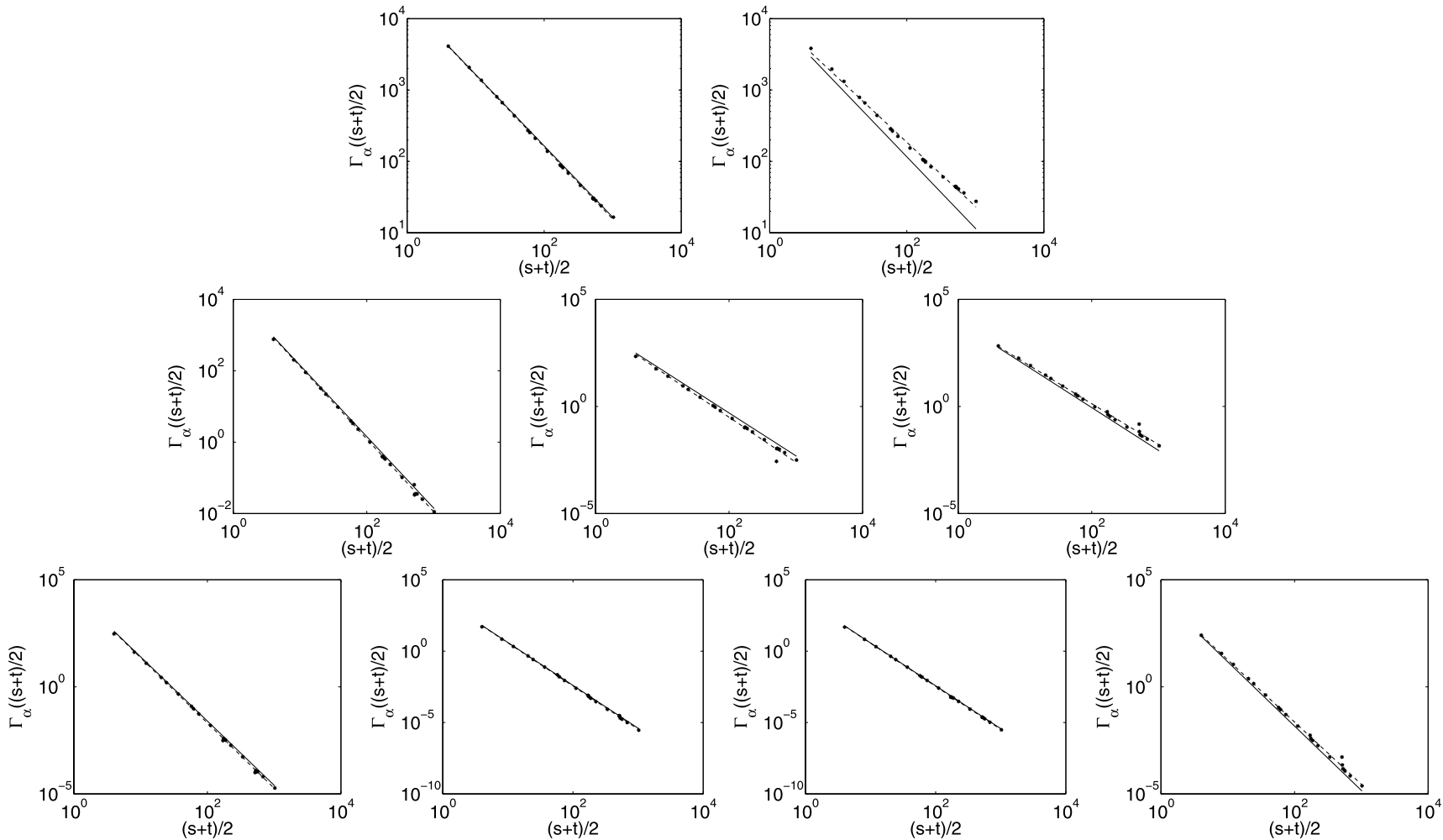

Fig. 2 Log-log plots of covariance estimates for natural image derivatives up to third order computed at scales $s, t$ from $2^{2}$ to $32^{2}$. In the reading direction we show results for $\alpha=$ $(1,0),(0,1),(2,0),(1,1),(0,2),(3,0),(2,1),(1,2),(0,3)$. The co-

Furthermore, if the image $f$ is assumed to be scale invariant, then we must recover the identity $s^{|\alpha|-H} \widehat{\Gamma}_{\alpha}(s)=$ $t^{|\alpha|-H} \widehat{\Gamma}_{\alpha}(t)$ within random variation.

In practice, the mapping $\xi: \mathbb{R}^{d} \times \mathbb{R}_{+} \rightarrow \mathbb{R}_{+}$usually is unknown. However, claiming stationarity of the increments of the image $f$, the above relations may be used for the estimation of $\xi$. Thus, the estimate $\widehat{\xi}$ should be such that $\widehat{\Gamma}_{\alpha}\left(\frac{s+t}{2}\right)$ does not depend on the spatial position $x$ and only depend on $s$ and $t$ through $s+t$, of course, again within random variation.

\subsection{Statistical Analysis of Natural Images}

In this section we investigate whether natural images have stationary increments and are scale invariant, i.e. whether we may choose $\xi(x, s)=s$. We compare with Lévy Brownian motion and a class of images with non-stationary increments.

We study the statistics of an ensemble of natural images consisting of 1630 images $(1024 \times 1536$ pixels) taken from the van Hateren natural stimuli collection [26]. For comparison, we generate two synthetic data sets of $1024 \times 1536$ pixel images: We sample 1630 Lévy Brownian images following the spectral representation proposed by Reed et al. variance estimates are shown as dots, the dashed light gray curve represents a power law $b[(s+t) / 2]^{-a}$ fitted to the estimates, and the fully drawn line shows the power law $b[(s+t) / 2]^{-|\alpha|}$

[23], by sampling standard Gaussian white noise in the Fourier domain and enforcing a power spectrum amplitude of $\|\omega\|^{-2-2 H}$, where $\omega$ denotes the spatial frequency vector and the Hurst index is $H=1 / 2$. And we sample 1630 sloped white noise images, constructed by adding i.i.d. standard Gaussian white noise to $f(x, y)=x^{4}$. The first synthetic data set has stationary increments, is isotropic and scale invariant, which is not the case for the latter data set. From each image we sample 1000 positions at random and compute the jet for $|\alpha| \leq 3$ at 6 different logarithmically sampled scales in the range $s \in\left[2^{2}, 32^{2}\right]$.

Figure 2 displays the estimates $\widehat{\Gamma}_{\alpha}\left(\frac{s+t}{2}\right)$ for $|\alpha| \leq 3$ taken at 6 scales which gives in total 36 estimates for each order $\alpha$. If the image has stationary increments, then these estimates should lie close to a single curve for each $\alpha$. Indeed we see in these log-log plots that the covariances seems to follow a power law which to some degree is proportional to $s^{-|\alpha|+H}$. Estimating $H$ from this power law fitted to the covariance estimates gives $H=0.006 \pm 0.072$ (the last number gives the standard deviation of the estimate). As a control experiment $H$ was estimated for the Lévy Brownian data set with the result $H=0.499 \pm 0.012$, which is within random variation of the theoretical value of $H=1 / 2$. This power law only seems to diverge for first order derivatives in the 

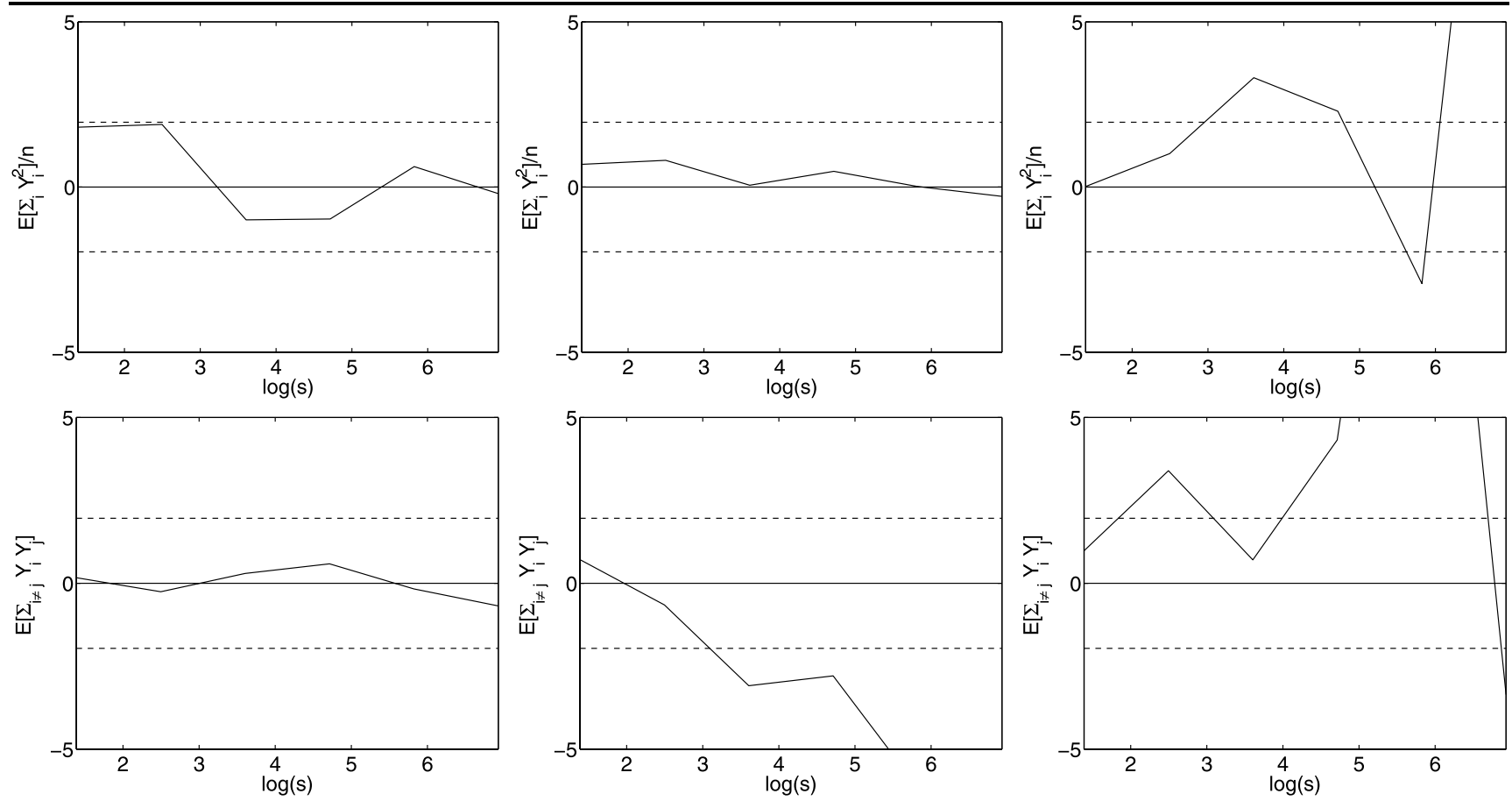

Fig. 3 Test statistics (5.5) for $E\left[\sum_{i} Y_{i}^{2}\right] / n-1=0$ (1st row) and $E\left[\sum_{i \neq j} Y_{i} Y_{j}\right]=0$ (2nd row) for Brownian images (1st column), natural images ( 2 nd column), and sloped white noise ( $3 r d$ column). The dotted lines display the acceptance region for the hypothesis test of stationarity on the $5 \%$-significance level

but this time using the slope relation $1 /(|\alpha|-H)$, and we get $H=0.123 \pm 0.132$. As a control experiment we also estimated $H$ on the Lévy Brownian data set, giving $H=0.473 \pm 0.036$ which is consistent with the theoretical $H=1 / 2$. The $H$ estimates from both Figs. 2 and 4 are summarized in Table 1.

The reason for the large standard deviation in the $\widehat{\Gamma}_{\alpha}(s) /$ $\sum_{\beta \in \mathbb{I}_{1}} \widehat{\Gamma}_{\alpha+\beta}(s)$ estimate, $H=0.123 \pm 0.132$, is due to a single outlier, corresponding to $\alpha=(0,1)$. The graph for $\alpha=(0,1)$ (see Fig. 4) is not following a line. The explanation for this is, as for Fig. 2, that the data set of natural images have a dominating horizon. Removing this outlier leads to an estimate of $H=0.068 \pm 0.050$, which is more consistent with the results for $\widehat{\Gamma}_{\alpha}\left(\frac{s+t}{2}\right)$ (Fig. 2), see Table 1.

To summarize the results, our ensemble of natural images seems to have stationary increments at low scales and the hypothesis seems to fail at larger scales. Furthermore, there are some indication that the hypothesis of isotropy only holds in a weak sense for this data set, appearing to break down for first order derivatives in the $y$-direction. The hypothesis of scale invariance as given by Definition 3 seems valid for our natural image data set. The estimate of the Hurst index for this data set is small $H \approx 0$. Field [3] find that on average across a limited set of six images depicting fairly irregular scenes the Hurst index is $H=0.5$ (based on orientation averaged power spectrum estimates $\|\omega\|^{-\gamma}$ and computed by $1 /(|\alpha|-H)$. We can again estimate the Hurst index $H$, 

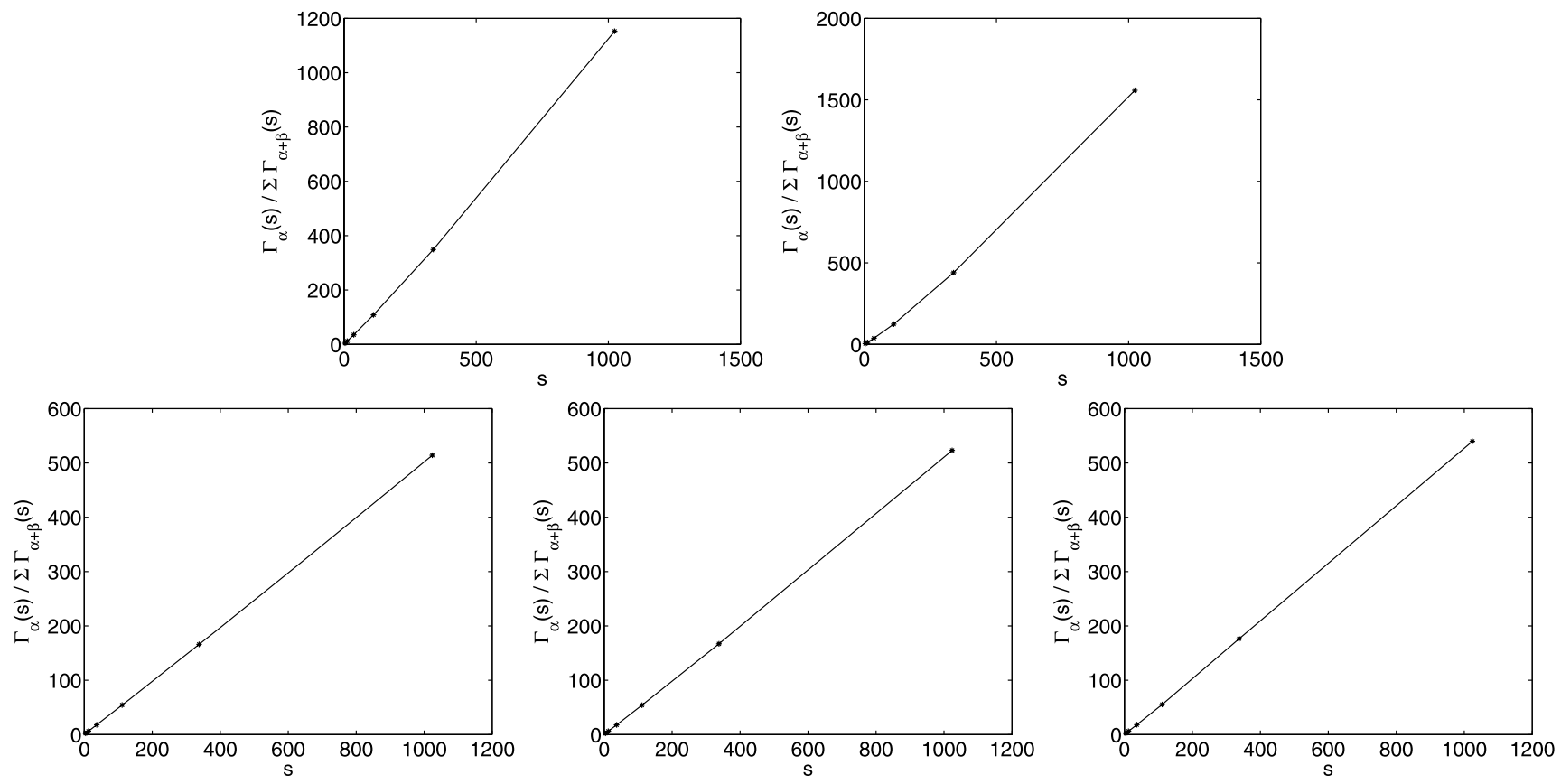

Fig. 4 Graphical test for scale invariance of natural image covariances. In the reading direction we show results for $\alpha=(1,0),(0,1),(2,0),(1,1),(0,2)$. The estimates are shown as dots with line segments in between

Table 1 Estimates of the Hurst index $H$ and standard deviations for natural images and Lévy Brownian images based on $\widehat{\Gamma}_{\alpha}\left(\frac{s+t}{2}\right)$ (Fig. 2) and $\widehat{\Gamma}_{\alpha}(s) / \sum_{\beta \in \mathbb{I}_{1}} \widehat{\Gamma}_{\alpha+\beta}(s)$ (Fig. 4). Last row shows the estimate of
$\widehat{\Gamma}_{\alpha}(s) / \sum_{\beta \in \mathbb{I}_{1}} \widehat{\Gamma}_{\alpha+\beta}(s)$ for natural images where $\alpha=(0,1)$ has been removed from the estimate

\begin{tabular}{llr}
\hline & Natural & Brownian \\
\hline$\widehat{\Gamma}_{\alpha}\left(\frac{s+t}{2}\right)$ & $H=0.006 \pm 0.072$ & $H=0.499 \pm 0.012$ \\
$\widehat{\Gamma}_{\alpha}(s) / \sum_{\beta \in \mathbb{I}} \widehat{\Gamma}_{\alpha+\beta}(s)$ & $H=0.123 \pm 0.132$ & $H=0.473 \pm 0.036$ \\
$\widehat{\Gamma}_{\alpha}(s) / \sum_{\beta \in \mathbb{I}} \widehat{\Gamma}_{\alpha+\beta}(s)$ without $\alpha=(0,1)$ & $H=0.068 \pm 0.050$ & - \\
\hline
\end{tabular}

using the relation $\gamma=2 H+1)$. Ruderman and Bialek [24] find a Hurst index of $H=0.405 \pm 0.005$ for a data set of images taken in the woods. Pedersen and Nielsen [19] find that on a limited set of 6 images containing a varying degree of clutter the Hurst index varies in the range $H=0.41$ to $H=0.97$. Balboa [2] compares power spectra for underwater scenes with atmospheric scenes and find that atmospheric scenes have power spectra corresponding to a Hurst parameter of $H=0.5 \pm 0.02$ and underwater scenes have $H=0.75 \pm 0.03$. The conclusion is that the Hurst index varies with image content and can to some extend be used to cluster images into different categories of content depending on the irregularity of, or clutter in, the image content.

Finally, we used the data set of Lévy Brownian images and the set of non-stationary sloped white noise images to validate the different test statistics presented in this section.

\section{Discussion and Conclusion}

We studied the second order structure of random images under the assumptions of stationary increments, isotropy and scale invariance. Scaling is defined through blurring with Gaussian kernels as described by linear scale space theory. The results presented provide constraints for the covariance structure of jets of scale space derivatives measured at a point.

The assumption of stationary increments dictates that the statistics of contrast changes in images remain the same irrespectively of position. This might not be true, e.g. think of a scene with varying global illumination such as sun light falling through a window into a room or long cast shadows. However, this assumption may be valid within regions of images and, as the results of Sect. 5 indicate, for small scales in ensembles of natural images with a large scene variation. In ensembles we see the full variation of statistics generated by the physics of light, e.g. the ensemble include both scenes 
with and without varying global illumination. Furthermore, such an ensemble will also include the effect that the camera was positioned randomly.

Isotropy implies that there are no preferred orientations in the studied images. However, this assumption is often violated, especially in images with a dominating horizon or in urban or indoor scenes where both horizontal and vertical orientations are dominating. This non-isotropy is amplified by the tendency for cameras to be oriented either nearly horizontal or vertical. This is also indicated by the results of Sect. 5, even though the non-isotropy is fairly weak. The assumption of isotropy will be valid for ensembles without dominating horizons and in general in scenes without dominating orientations, which rules out most urban and indoor scenes. But the assumption may still be used as an a priori constraint in general purpose image analysis systems as the least committed assumption on orientations. Hence it will be the least committed choice, e.g. if our camera can be randomly oriented.

Scale invariance is a fundamental property found in abundance in nature and it is reasonable to assume that such invariance property also holds for images of naturally occurring scenes. An image is a measurement of light intensities captured at a certain resolution given by the size of the pixels and with a certain limited field of view. The scenes measured might also have a lower limit of scales of objects visible in the image which can be larger than the pixel scalethis scale is sometimes referred to as the inner scale. Similarly the limited field of view provides the upper scale-also known as the outer scale - but the measured scene may only include objects up to a certain size which effectively lowers the outer scale. Hence, we can only expect to see the scale invariance property in natural images within a range of scales given by the inner and outer scales. Indeed the results of Sect. 5 indicate that scale invariance is present in our ensemble of natural images.

Natural images are not completely characterized by their covariance structure, however the studied class of models may act as prior models in various vision and image processing tasks, a few of which was mentioned in the introduction. We believe that the studied models will be useful in other areas of vision and image research, e.g. as prior models of local structure in problems such as denoising, deconvolution and inpainting or image completion problems, but this still remains to be seen.

Acknowledgements This research was partly funded by the Danish Natural Science Research Council framework grant Natural Image Sequence Analysis (FNU 272-05-0256).

\section{References}

1. Attneave, F.: Some informational aspects of visual perception. Psychol. Rev. 61, 183-193 (1954)
2. Balboa, R.M.: Power spectra and distribution of contrasts of natural images from different habitats. Vis. Res. 43(24), 2527-2537 (2003)

3. Field, D.J.: Relations between the statistics of natural images and the response properties of cortical cells. J. Opt. Soc. Am. 4(12), 2379-2394 (1987)

4. Florack, L.M.J.: Image Structure. Computational Imaging and Vision. Kluwer Academic, Dordrecht (1997)

5. Griffin, L.D.: The second order local-image-structure solid. IEEE Trans. Pattern Anal. Mach. Intell. 29(8), 1355-1366 (2007)

6. Iijima, T.: Basic theory of pattern observation. Technical report, Papers on technical group on automata and automatic control (1959), in Japanese

7. Iijima, T.: Theory of pattern recognition. Electron. Commun. Jpn. 123-134 (1963)

8. Koenderink, J.J.: The structure of images. Biol. Cybern. 50, 363370 (1984)

9. Koenderink, J.J., van Doorn, A.J.: Representation of local geometry in the visual system. Biol. Cybern. 55, 367-375 (1987)

10. Koenderink, J.J., van Doorn, A.J.: Local structure of Gaussian texture. J. Inst. Electron. Inf. Commun. Eng. Trans. Inf. Syst. E86D(7), 1165-1171 (2003)

11. Longuet-Higgins, M.S.: The statistical analysis of a random, moving surface. Philos. Trans. R. Soc. Lond. Ser. A Math. Phys. Sci. 249(966), 321-387 (1957)

12. Longuet-Higgins, M.S.: Statistical properties of an isotropic random surface. Philos. Trans. R. Soc. Lond. Ser. A Math. Phys. Sci. 250(975), 157-174 (1957)

13. Loog, M., Pedersen, K.S., Markussen, B.: Maximum likely scale estimation. In: Olsen, F.O., Floruck, L.M.J. Kuipjer, A. (eds.) Deep Structure, Singularities and Computer Vision. LNCS, vol. 3753, pp. 146-156. Springer, Berlin (2005)

14. Mandelbrot, B.B., van Ness, J.W.: Fractional Brownian motions, fractional noises and applications. SIAM Rev. 10(4), 422-437 (1968)

15. Markussen, B., Pedersen, K.S., Loog, M.: A scale invariant covariance structure on jet space. In: Olsen, F.O., Florack, L.M.J. Kuijper, A. (eds.) Deep Structure, Singularities and Computer Vision. LNCS, vol. 3753, pp. 12-23. Springer, Berlin (2005)

16. Markussen, B., Sporring, J., Erleben, K.: Guessing tangents in normal flows. J. Math. Imag. Vis. (2008, this issue)

17. Masry, E.: The wavelet transform of stochastic processes with stationary increments and its application to fractional Brownian motion. IEEE Trans. Inf. Theory 39(1), 260-264 (1993)

18. Pedersen, K.S.: Properties of Brownian image models in scalespace. In: Griffin, L.D., Lillholm, M. (eds.) Proceeding of the 4th Scale-Space Conference. LNCS, vol. 2695, pp. 281-296. Springer, Berlin (2003)

19. Pedersen, K.S., Nielsen, M.: The Hausdorff dimension and scalespace normalisation of natural images. J. Vis. Commun. Image Represent. 11(2), 266-277 (2000)

20. Pedersen, K.S., Loog, M., Markussen, B.: Generic maximum likely scale selection. In: 1st International Conference on Scale Space and Variational Methods in Computer Vision. LNCS, vol. 4485, pp. 362-373. Springer, Berlin (2007)

21. Pedersen, K.S., Loog, M., van Dorst, P.: Salient point and scale detection by minimum likelihood. In: JMLR: Workshop and Conference Proceedings: Gaussian Processes in Practice, vol. 1, pp. 59-72 (2007)

22. Pesquet-Popescu, B.: Statistical properties of the wavelet decomposition of certain non-Gaussian self-similar processes. Sign. Process. 75(3), 303-322 (1999)

23. Reed, I.S., Lee, P.C., Truong, T.K.: Spectral representation of fractional Brownian motion in $\mathrm{n}$ dimensions and its properties. IEEE Trans. Inf. Theory 41(5), 1439-1451 (1995) 
24. Ruderman, D.L., Bialek, W.: Statistics of natural images: scaling in the woods. Phys. Rev. Lett. 73(6), 814-817 (1994)

25. Srivastava, A., Lee, A.B., Simoncelli, E.P., Zhu, S.-C.: On advances in statistical modeling of natural images. J. Math. Imaging Vis. 18(1), 17-33 (2003)

26. van Hateren, J.H., van der Schaaf, A.: Independent component filters of natural images compared with simple cells in primary visual cortex. Proc. R. Soc. Lond. Ser. B 265, 359-366 (1998)

27. Witkin, A.P.: Scale space filtering. In: Proc. of the Eighth International Joint Conference on Artificial Intelligence, vol. 2, pp. 10191023. Karlsruhe, Germany (1983)

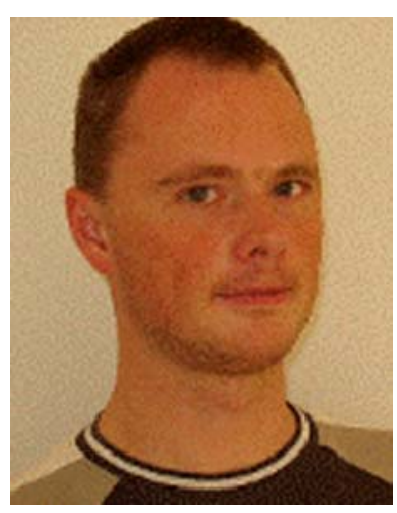

Bo Markussen received the Cand. Scient and Ph.d. degrees in mathematical statistics from University of Copenhagen, Denmark. From November 2001 to September 2003 he worked as post doc in Copenhagen and Berlin, and from October 2003 to December 2005 he was enrolled in the "Computing Natural Shape" project at the Department of Computer Science, University of Copenhagen. In January 2006 he joined the Deparment of Natural Sciences at the Royal Veterinary and Agricultural University (KVL), Denmark, as an Assistent Professor of statistics. KVL has now been merged into the University of Copenhagen as the Faculty of Life Sciences. The research interests of Bo Markussen includes applied statistics, stochastic calculus and applications to image analysis.

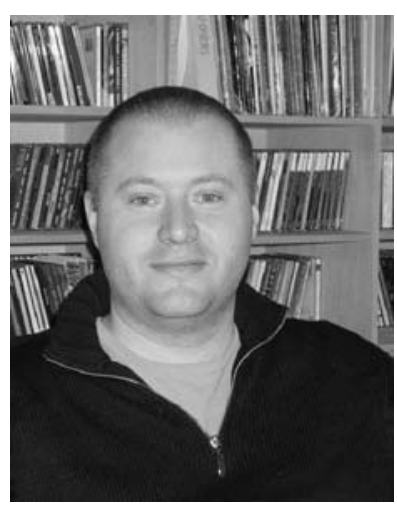

Kim Steenstrup Pedersen received a M.Sc. in 1999 and a Ph.D. in 2003 both in computer science from DIKU, Department of Computer Science, University of Copenhagen, Denmark and he also holds a B.Sc. degree in physics from the University of Copenhagen. During his $\mathrm{Ph} . \mathrm{D}$. studies he spent half a year in 2001 at Division of Applied Mathematics, Brown University, Rhode Island, USA. After his Ph.D. he was briefly employed as an assistant research professor at DIKU. From spring 2003 to 2006 he was assistant professor at the IT University of Copenhagen, Denmark. He currently holds a position as associate professor at DIKU, Department of Computer Science, University of Copenhagen. His primary research interests include topics from computer vision and image analysis, especially scale-space theories, natural image statistics, stochastic image models, and image features.

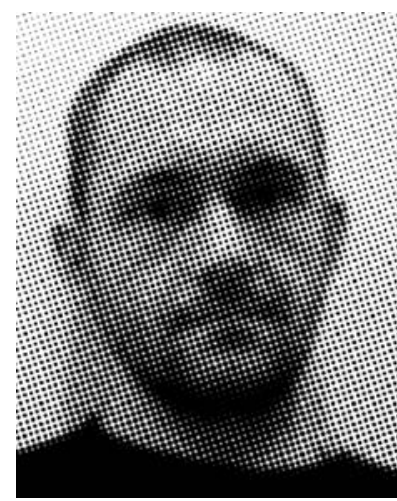

Marco Loog received an M.Sc. degree in mathematics from Utrecht University and a Ph.D. degree from the Image Sciences Institute. At present, he is an assistant professor at Delft University of Technology with a secondary affiliation to the University of Copenhagen. His everevolving research interests currently include man and machine saliency, probabilistic scale space theory, folklore theorems, black math, procrustean discriminant analysis, and dissimilarity-based pattern recognition. Dr. Loog is an advocate of the lost art of critical appraisal. His research is supported by the Innovational Research Incentives Scheme of the Netherlands Research Organization [NWO] and the Research Grant Program of the Faculty of Science, University of Copenhagen. 